\title{
Obtención de leche bovina reducida en grasa saturada y naturalmente enriquecida en ácido linoleico conjugado
}

\author{
Production of bovine milk reduced in saturated fat \\ and naturally enriched in conjugated linoleic acid
}

Gagliostro, Gerardo. A. (1); Antonacci, Liliana. E. (1); Carabajal, Augusto (2); López Plaván, Juan. M. (2); Crujeira, Yolanda (2)

(1) INTA EEA Balcarce, Argentina.

(2) Complejo Agroindustrial Talar, Laguna del Sauce, Punta del Este, Uruguay.

Contacto: gagliostro.gerardo@inta.gob.ar

RECIBIDO: $12 / 3 / 2018$ - APROBADO: $1 / 6 / 2018$

\begin{abstract}
Resumen
El objetivo del trabajo fue reducir la presencia de los ácidos grasos (AG) saturados potencialmente aterogénicos (C12:0, C14:0 y C16:0) de la leche y aumentar la presencia del ácido linoleico conjugado (CLA) en vacas lecheras estabuladas alimentadas con ración completamente mezclada (RCM) a través del aporte suplementario de ácidos grasos poliinsaturados (AGPI). Se utilizaron dos lotes de 70 vacas multíparas en el primer tercio de lactancia. Luego de seis semanas experimentales y durante cinco días consecutivos se obtuvieron cinco muestras de leche estándar (LE) provenientes del tanque colector del lote vacas sin AGPI suplementarios, y cinco muestras de leche CLA (LCLA) provenientes del tanque de las vacas suplementadas con los AGPI. El tenor graso (g/100g) de la LE $(3,84)$ resultó superior $(\mathrm{p}<0,001)$ al de LCLA $(2,84)$ por reducción $(17,21 \%)$ de los AG potencialmente aterogénicos. El índice aterogénico fue mayor $(\mathrm{p}<0,001)$ en la LE $(4,07)$ comparado al de la LCLA $(2,58)$. La concentración del CLA aumentó $(\mathrm{p}<0,001)$ un $122 \%$ en LCLA $(0,77 \mathrm{~g} / 100 \mathrm{~g})$ respecto al valor registrado en LE $(0,34 \mathrm{~g} / 100 \mathrm{~g})$. El ácido oleico $(\mathrm{g} / 100 \mathrm{~g})$ resultó superior $(\mathrm{p}<0,001)$ en la LCLA $(23,37)$ comparado con la LE $(18,78)$. El aporte suplementario de AGPI permitió incrementar el valor saludable de la leche producida en un sistema de alimentación estabulado manteniendo una adecuada relación $(6,59)$ omega-6/omega-3 en la leche y confirmando la plasticidad en la composición de los AG constitutivos de la grasa láctea.

Palabras clave: Vaca lechera, alimentación estabulada, perfil de la grasa láctea.
\end{abstract}

\begin{abstract}
The aim of the work was to reduce the presence of the potentially atherogenic fatty acids (FA) of milk (C12: 0 , C14: 0 and C16: 0) and increase the presence of conjugated linoleic acid (CLA) in confined dairy cows fed a total mixed ration (TMR) supplemented with polyunsaturated fatty acids (PUFA). Two batches of 70 multiparous cows in the first third of lactation were used. After six experimental weeks and for five consecutive days, milk samples (5) were obtained from the collector tank of non-supplemented cows (LE) and of CLA milk (LCLA) from the tank of PUFA supplemented cows. Fat content $(\mathrm{g} / 100 \mathrm{~g})$ in LE $(3,84)$ was higher $(\mathrm{p}<0,001)$ than LCLA $(2,84)$ explained by a significant reduction $(17,21 \%)$ in the concentration of the potentially atherogenic FA of milk. The milk atherogenic index was higher $(\mathrm{p}<0,001)$ in LE $(4,07)$ compared to LCLA $(2,58)$. Milk CLA content increased $(\mathrm{p}<0,001)$ by $122 \%$ in LCLA $(0,77 \mathrm{~g} / 100 \mathrm{~g}$ FA) compared to LE $(0,34 \mathrm{~g} / 100 \mathrm{~g}$ FA). Oleic acid concentration ( $\mathrm{g} / 100$ $\mathrm{g} F A)$ resulted higher $(\mathrm{p}<0,001)$ in LCLA $(23,37)$ compared to LE $(18,78)$. Supplementation with PUFA in confined dairy cows increased the healthy value of the milk produced maintaining an adequate omega-6/omega- 3 ratio $(6,59)$ and confirmed the plasticity in milk fatty acid composition.

Keywords: Dairy cow, confined production system, milk fatty acid profile.
\end{abstract}

\section{Introducción}

La creciente incidencia en la sociedad moderna de enfermedades cardiovasculares (ECV) y crónicas no transmisibles (ECNT) implica una importante carga económica y social para los estados y genera la necesidad de producir alimentos más saludables capaces de atenuar esos riesgos. La leche y sus derivados han sido blanco de críticas ya que representan la mayor contribución al consumo de AG saturados (AGS) en el ser humano (Chilliard, et al., 2000; Chilliard y Ferlay, 2004) y, en consecuencia, la ingestión de lácteos ha sido desvalorizada (Siurana y Calsamiglia, 2016). Sin embargo, numerosos estudios sugieren que la relación entre el consumo de AGS con la aterosclerosis y las ECV resulta a veces inconsistente y hasta 
contradictoria (Siurana y Calsamiglia, 2016). Recientes trabajos afirman que el consumo de lácteos reduce la incidencia de ECV a pesar de su exceso en grasa saturada y atribuyen parte del efecto cardioprotector a la presencia del isómero cis-9, trans-11 del ácido linoleico llamado ácido linoleico conjugado natural o CLA (Smit, et al., 2010). Otros compuestos presentes en los lácteos (calcio, péptidos bioactivos, ácido fólico y vitaminas) son también saludables y contribuyen a potenciar el efecto (Siurana y Calsamiglia, 2016). El CLA natural está presente únicamente en carnes y leches de rumiantes (Siurana y Calsamiglia, 2016; Smit, et al., 2010), y la leche es la principal fuente de este AG saludable (Chilliard, et al., 2007, Smit, et al., 2010). A partir del análisis de nueve estudios en humanos, Livingstone et al. (2012) concluyeron que el consumo crónico de leche con un perfil en AG modificado resultó beneficioso para la salud cardiovascular en individuos normales e hipercolesterolémicos. Por su parte, Smit et al. (2010) reportaron que una concentración alta de CLA utilizado como biomarcador en tejido adiposo humano como consecuencia del consumo de leche rica en CLA, estuvo asociada a un menor riesgo de infarto de miocardio en una población de 1813 casos de primer infarto agudo no fatal y 1813 individuos control agrupados por edad, sexo y área de residencia. Según estos autores, el CLA natural puede ventajosamente contrarrestar el riesgo de consumir en exceso la grasa saturada presente en los lácteos (Chilliard, et al., 2000) sin riesgo sobre infarto agudo de miocardio. En adición a los efectos cardio protectores del CLA (Chilliard, et al., 2000; Roy, et al., 2017; Livingstone, et al., 2012), estudios con animales o cultivos celulares con diferentes líneas tumorales han demostrado promisorios efectos anticáncer (Chilliard, et al., 2000; Bhattacharya, et al., 2006; De La Torre, et al., 2006; Larson, et al., 2005). El consumo de lácteos estándar no permitiría alcanzar la ingestión diaria de CLA necesaria para la expresión de sus potenciales efectos preventivos de patologías cardiovasculares (Bhattacharya, et al., 2006), diabetes (Ryder, et al., 2001; Houseknecht, 1998), aterosclerosis (Lee, et al., 1994), cáncer (Ha, et al., 1990; Ip, et al., 1999), hipertensión (Bhattacharya, et al., 2006) y obesidad (Watkins y Li, 2003; Bhattacharya, et al., 2006). También se describen efectos antiinflamatorios del CLA (Yang y Cook, 2003; Miller, et al., 1994).

La leche bovina es la principal fuente natural del CLA, pero la producida en sistemas de alimentación estabulados sin acceso a pastoreo directo se caracteriza por bajos contenidos de CLA y un exceso de grasa saturada (Dhiman, et al., 2005). Consumidos en exceso, los AGS no saludables de la leche serían el láurico (C12:0), el mirístico (C14:0) y el palmítico (C16:0) por su potencial rol aterogénico (Ulbritch y Southgate, 1991) y por estar asociados al riesgo de ECV (Grummer, 1991; Stanton, et al., 2003). Aunque el efecto no saludable de los AGS de cadena media es aún controversial, su consumo excesivo puede prevenirse reduciendo en forma natural la presencia en leche de los mismos a través de la alimentación estratégica del ganado lechero (Chilliard, et al., 2000; Chilliard y Ferlay, 2004), mejorando la imagen y el valor saludable de los lácteos.

La suplementación con AG poliinsaturados (AGPI) en vacas lecheras permite modificar la composición en AG de la leche y sus derivados en un sentido favorable a la salud humana (Chilliard, et al., 2000; Chilliard, et al., 2007). El objetivo de este trabajo fue conocer el efecto del aporte de un suplemento lipídico con AGPI sobre el perfil en AG de la leche bovina producida en un sistema de alimentación estabulado sin acceso a forraje fresco.

\section{Materiales y Métodos}

El trabajo se llevó a cabo en el complejo agroindustrial Talar, situado en la localidad de Laguna del Sauce, Ruta 12, km 10, Maldonado (Uruguay). El establecimiento cuenta con vacas de raza Holstein alimentadas en un sistema estabulado sin acceso a pastoreo directo. Se utilizaron 70 vacas multíparas alimentadas con una ración completamente mezclada (RCM) sin lípidos suplementarios para obtención de la leche estándar (LE) y 70 vacas productoras de leche CLA (LCLA) que consumieron la misma RCM con el agregado de $0,7 \mathrm{~kg} / \mathrm{vaca} /$ día de un suplemento lipídico comercial ( $90 \%$ de materia seca (MS) y $68,6 \%$ de grasa) con 10,3\% de ácido palmítico (C16:0), 3,9\% de ácido esteárico (C18:0), 24,9\% de ácido oleico (C18:1), 45,6\% de ácido linoleico (C18:2), 4,8\% de ácido linolénico (C18:3), 0,6\% de ácido eicosapentaenoico (EPA, $\mathrm{C}_{20: 5}$ y $1 \%$ de ácido docosahexaenoico (DHA, $\mathrm{C}_{22: 6}$ ). El suplemento fue incorporado a la RCM sin acostumbramiento previo de los animales.

Las vacas produjeron en promedio 35 litros/vaca.día y se encontraban en el primer tercio de lactancia ( $<100$ días posparto). En alimento tal cual, la RCM estuvo compuesta por ensilaje de planta entera de maíz planta $(45,09 \%)$, ensilaje de raigrás $(20,89 \%)$, heno de raigrás $(1,7 \%)$, suero de leche $(9,3 \%)$, grano de maíz molido $(9,81 \%)$, harina de soja $(5,03 \%)$, expeller de soja $(5,58 \%)$, harina de trigo $(1,3 \%)$ y un núcleo $(1,3 \%)$ vitamínico-mineral de uso habitual en el tambo (Nutral).

Luego de seis semanas de alimentación y durante cinco días consecutivos, se obtuvieron cinco muestras diarias (100 mL) de leche del tanque colector de la LE y del tanque colector de la LCLA. En cada día de muestreo y para cada muestra de tanque $(100 \mathrm{~mL})$ se separaron $20 \mathrm{~mL}$ para las determinaciones de composición química (grasa butirosa, proteína, lactosa, sólidos totales y sólidos no grasos) en el Laboratorio de Calidad de Leche del Instituto Nacional de Investigación Agropecuaria (INIA). Los $80 \mathrm{~mL}$ restantes fueron inmediatamente congelados $\left(-20^{\circ} \mathrm{C}\right)$ para el ulterior análisis del perfil en AG por cromatografía gas-líquido (GLC), según se describe en Masson et al. (2015). Las diferencias de composición de leche y perfil de AG entre lotes (LE vs LCLA) fueron analizadas a través del test $\mathrm{T}$ de Student para observaciones independientes.

\section{Resultados y Discusión}

La composición química de la leche estándar (LE) y de la leche CLA (LCLA) se presenta en la Tabla 1.

El tenor graso de la leche resultó menor $(-0,96 \mathrm{~g} / 100 \mathrm{~g})$ en LCLA, lo que significó un $25,08 \%$ de disminución respecto a la LE. La inhibición de la síntesis de novo mamaria con la correspondiente reducción en la concentración de AGS en la leche (Tabla 2) explica la caída en el tenor graso. En vacas lecheras en alimentación base pastoril el suministro de lípidos insaturados suele reducir el contenido de grasa butirosa de la leche en un $8 \%$ en promedio (Schroeder, et al., 2004), resultado inferior al obtenido en este ensayo (25\%). La presencia en el suplemento utilizado del DHA (inhibidor de la lipogénesis mamaria de novo), más la generación de ciertos AG como el trans-10 $\mathrm{C}_{18: 1}$ y su posterior transferencia a la leche (Tabla 2), explicarían la importante disminución (Chilliard, et al., 2000). Se ha informado una relación directa entre niveles crecientes de trans-10 C18:1 en leche y reducción de la síntesis mamaria 


\begin{tabular}{|l|c|c|c|c|}
\hline Parámetro & LE & LCLA & p $<(1)$ & $\Delta \%(2)$ \\
\hline Grasa butirosa, g/100g & $3,84( \pm, 15)$ & $2,88( \pm 0,23)$ & $* * * *$ & $-25,08$ \\
Proteína, g/100g & $3,39( \pm 0,05)$ & $3,32( \pm 0,10)$ & $* * * *$ & $-2,33$ \\
Lactosa, g/100g & $5,04( \pm 0,04)$ & $4,95( \pm 0,11)$ & $* * * *$ & $-1,73$ \\
Grasa/Proteína & $1,13( \pm 0,04)$ & $1,01( \pm 0,14)$ & $* * * *$ & $-10,67$ \\
Sólidos totales, g/100g & $13,05( \pm 0,20)$ & $12,40( \pm 0,71)$ & $* * * *$ & $-4,97$ \\
\hline
\end{tabular}

(1) ${ }^{* * * *}$ Probabilidad significativa $(\mathrm{p}<0,001$, Test $\mathrm{t}$ de Student).

(2) Variación porcentual respecto al lote estándar. Los resultados se expresan a través del promedio \pm el desvío estándar.

Tabla 1. Composición química de la leche de vacas alimentadas con la ración control (LE) y de las suplementadas con ácidos grasos poliinsaturados (LCLA).

\begin{tabular}{|c|c|c|c|c|}
\hline Ácido Graso (g/100 g de AG) & LE & LCLA & p $<(2)$ & $\Delta \%(3)$ \\
\hline C12:0 & $3,80( \pm 0,13)$ & $2,73( \pm 0,19)$ & 0,00001 & $-28,19$ \\
C14:0 & $12,71( \pm 0,07)$ & $11,26( \pm 0,32)$ & 0,00001 & $-11,42$ \\
C16:0 & $36,43( \pm 2,97)$ & $29,84( \pm 0,70)$ & 0,00001 & $-18,08$ \\
LC12:0-C16:0 & $52,94( \pm 0,27)$ & $43,83(1,15)$ & 0,00001 & $-17,21$ \\
C18:0 & $8,11( \pm 0,05)$ & $10,77( \pm 1,20)$ & 0,00001 & $+32,81$ \\
trans-9 C18:1 & $0,09( \pm 0,03)$ & $0,29( \pm 0,06)$ & 0,0002 & +211 \\
trans-10 C18:1 & $0,23( \pm 0,02)$ & $1,05( \pm 0,24)$ & 0,00001 & +358 \\
trans-11 C18:1, AV & $0,54( \pm 0,04)$ & $1,30( \pm 0,16)$ & 0,00001 & $+138,6$ \\
cis-9 C18:1 & $18,78( \pm 0,08)$ & $23,37( \pm 0,61)$ & 0,00001 & $+24,43$ \\
cis-9,cis-12 C18:2 & $1,92( \pm 0,04)$ & $2,51( \pm 0,14)$ & 0,00001 & $+30,83$ \\
cis-9,trans-11 C18:2 (CLA) & $0,34( \pm 0,01)$ & $0,76( \pm 0,10)$ & 0,00001 & +122 \\
cis-9,12, 15 C18:3 & $0,29( \pm 0,01)$ & $0,38( \pm 0,03)$ & 0,0001 & $+32,64$ \\
IA ${ }^{(4)}$ & $4,07( \pm 0,03)$ & $2,58(0,18)$ & 0,00001 & $-36,75$ \\
Relación n 6/n 3 & $6,67( \pm 0,10)$ & $6,59( \pm 0,29)$ & 0,57 & Sin efecto \\
\hline
\end{tabular}

(1) Los resultados se expresan a través del promedio \pm el desvío estándar $(\mathrm{n}=5)$.

(2) Probabilidad estadística de la diferencia entre promedios.

(3) Diferencia porcentual respecto al Lote Estándar.

(4) Índice de aterogenicidad.

Tabla 2. Composición en ácidos grasos de interés de la leche producida por las vacas del lote estándar (LE) y de la leche del lote CLA (LCLA) (1)

de novo (Piperova, et al., 2000), lo que contribuye a explicar la caída en el tenor graso. Una alta concentración de trans-10 $\mathrm{C}_{18: 1}$ ha sido asociada a disfunciones en la actividad de las enzimas lipoproteína lipasa (LPL) y estearil CoA desaturasa (SCD) que intervienen en la síntesis de grasa causando así una disminución en el tenor graso de la leche (Griinari y Bauman, 1999). En este trabajo la reducción en la concentración grasa se produjo a expensas de los AG potencialmente hipercolesterolémicos, lo que contribuye a disminuir el índice aterogénico de la leche (Tabla 2).
La diferencia en el tenor proteico de la leche del lote CLA resultó muy leve $(-0,08 \mathrm{~g} / 100 \mathrm{~g})$ respecto a la LE, lo que implicó un 2,33\% de disminución (Tabla 1). La relación grasa/proteína resultó cercana a 1, y por ende adecuada para la elaboración de quesos a partir de leche CLA. En condiciones de alimentación pastoril, la suplementación con lípidos no suele afectar la concentración proteica de la leche (Bargo, et. al., 2003; Schroeder, et al., 2004), mientras que en alimentación estabulada este parámetro resulta sistemáticamente afectado (Gagliostro y Chilliard, 1992; Wu y Huber, 1994). El efecto negativo sobre 
la concentración de proteína en la leche de vacas (Gagliostro y Chilliard, 1992; Palmquist, et al., 1993) resulta más consistente ante el uso de grasas saturadas $(-0,18 \mathrm{~g} / 100 \mathrm{~g})$ y sales cálcicas de aceite de palma $(-0,12 \mathrm{~g} / 100 \mathrm{~g})$ respecto a los aceites vegetales poliinsaturados (Gagliostro y Chilliard, 1992).

La concentración de lactosa resultó ligeramente menor en la leche CLA $(-0,09 \mathrm{~g} / \mathrm{kg}$, Tabla 1$)$, lo que puede juzgarse como positivo en casos de intolerancia a la lactosa o desde el punto de vista industrial (menor acidez en quesos). Finalmente, la concentración de sólidos totales fue menor en la leche CLA $(-0,65 \mathrm{~g} / 100 \mathrm{~g})$ como consecuencia de la menor concentración grasa observada en este lote.

\section{Perfil en ácidos grasos de la leche}

Si bien se dispone de un perfil completo de los AG constitutivos, a los fines de este trabajo se presentan y discuten solo aquellos que resultan más relevantes para mejorar el valor saludable o funcional de la leche (Tabla 2).

Respecto a la LE, la LCLA presentó una importante reducción $(-17,21 \%)$ en la concentración total de los AGS potencialmente aterogénicos (C12:0 a C16:09), cuyo consumo excesivo resulta prudente evitar (Ulbritch y Southgate, 1991). Expresada en valor absoluto, la reducción fue del orden de $9,11 \mathrm{~g} / 100 \mathrm{~g}$ de AG totales y satisface el criterio de funcionalidad que menciona la reducción de un componente o componentes no saludables. La concentración en LE (12,71 $\mathrm{g} / 100 \mathrm{~g}$ ) del ácido mirístico (C14:0), cuyo rol pro-aterogénico fue considerado muy potente (Ulbritch y Southgate, 1991), se redujo a 11,26 g/100 g en la LCLA. Expresadas en forma porcentual respecto a valores de la $\mathrm{LE}$, las reducciones fueron de $28,19 \%$ para el C12:0, 11,42\% para el C14:0 y del 18,08\% para el C16:0 (Tabla 2). El consumo excesivo de estos tres AGS podría elevar el colesterol plasmático total y el colesterol asociado a las lipoproteínas plasmáticas (LDL) de baja densidad (Schrezenmeir y Jagla, 2000). En consecuencia, estos resultados mejoran el valor nutricional de la LCLA.

La reducción en la concentración de estos AGS como consecuencia del consumo de AGPI como los contenidos en el suplemento utilizado es un efecto bien documentado (Chilliard, et al., 2000; AbuGhazaleh y Jenkins, 2004; Zheng, et al., 2005; Huang, et al., 2008) que se explicaría por la producción a nivel de rumen de ciertos AG trans como el trans-10 C18:1 por biohidrogenación de los AGPI. Estos AG trans resultan potentes inhibidores de enzimas clave de la lipogénesis mamaria, como la acetil-CoA carboxilasa (Chilliard, et al., 2000; Chilliard y Ferlay, 2004). Como resultado asociado, el IA de la leche disminuyó desde un valor de 4,07 en la LE a uno de 2,58 en la LCLA. Esto implicó un 36,75\% de reducción de este parámetro asociado al riesgo cardiovascular de origen dietario. Resultados previos también indicaron disminuciones en el IA de la leche desde un valor basal de 1,88 a $0,5-0,8$ en vacas lecheras que recibieron aceites de girasol y de pescado (Gagliostro, et al., 2017). Estos resultados contribuyen a evitar un exceso en el consumo de AGS no saludables y, potencialmente, a disminuir la presencia de marcadores sanguíneos asociados al riesgo cardiovascular.

La concentración en LE de los AG trans-9 C18:1 (0,09 $\mathrm{g} / 100 \mathrm{~g})$ y trans-10 C18:1 (0,23 g/100 g de AG) resultaron normales y compatibles con los elevados valores de concentración de grasa láctea obtenidos $(3,84 \mathrm{~g} / 100 \mathrm{~g}$, Tabla 1). Tanto las concentraciones del trans- $9 \mathrm{C} 18: 1$ como la del trans-10 C18:1 fueron incrementadas en la LCLA y alcanzaron registros promedio de $0,29 \mathrm{y}$ de $1,05 \mathrm{~g} / 100 \mathrm{~g}$, respectivamente (Tabla 2). Estos valores pueden considerarse inocuos en salud humana o sin riesgos sobre el grado de cardiopatía isquémica en seres humanos (Roy, et al., 2007). La presencia del DHA en el suplemento utilizado pudo haber contribuido a mantener niveles bajos del trans-10 $\mathrm{C}_{18: 1}$, ya que la concentración de este isómero en la leche tendió a disminuir ante la participación creciente del aceite de pescado (fuente natural de DHA y EPA) en mezcla con aceite de girasol (Palmquist y Griinari, 2006).

La concentración del ácido vaccénico (trans-11 C18:1, AV) en la LCLA fue de 1,30 g/100 g de AG, lo que representó un $138,6 \%$ de aumento respecto al valor de $0,54 \mathrm{~g} / 100 \mathrm{~g}$ de AG registrado en la LE. El AV representó el 62,79\% del total de los AG trans-C18:1 en la leche Control, resultado bajo en relación al $80,41 \%$ esperable y a lo reportado por Antonacci et al. (2017). Dicha proporción disminuyó en la leche CLA a valores de $49,24 \%$, mientras que en vacas en sistema pastoril suplementadas con aceites de soja y lino la proporción permaneció elevada (77 a 82\%), con proporciones de trans-9 y trans-10 C18:1 de tan solo 11,5 y 28,9\%, respectivamente (Antonacci, et al., 2017). En este trabajo, la proporción del trans -9 resultó baja (Control $=10,47 \%$ y CLA $=10,98 \%$ ) pero la del trans-10 C18:1 experimentó un importante aumento en el tratamiento CLA (Control $=26,74 \%$ y CLA=39,77). Estos resultados podrían indicar una actividad subóptima de biohidrogenación ruminal por parte de bacterias clave como el Butivibrio fibrisolvens inducida por el tipo de ración, por alguno de sus componentes y/o por algún factor desconocido, lo que limita los objetivos de obtener una leche con mayor contenido de CLA. Un desvío hacia la formación de trans-10 C18:1 también explicaría en parte el resultado obtenido (Tabla 2).

$\mathrm{El} \mathrm{AV}$ presente en los lácteos puede otorgar propiedades beneficiosas a través de un efecto anticarcinogénico directo (Awad, et al., 1995), o mediado por su conversión endógena al CLA a una tasa estimada en un 20\% (Turpeinen, et al., 2002) por acción de la enzima $\Delta 9$ desaturasa en el ser humano (Chilliard, et al., 2000; Parodi, 2003). La metabolización del AV a CLA ha demostrado ser efectiva en la prevención del cáncer químicamente inducido en ratas (Banni, et al., 2001) e incrementa la biodisponibilidad del compuesto en los tejidos (Chilliard, et al., 2000; Siurana y Calsamiglia, 2016). Por las razones expuestas, el incremento obtenido fue moderado (Tabla 2) y deberá potenciarse.

La concentración del CLA (Tabla 2) se incrementó en un $122 \%$ al comparar el valor en la LE $(0,34 \mathrm{~g} / 100 \mathrm{~g})$ al observado en la LCLA $(0,77 \mathrm{~g} / 100 \mathrm{~g})$. Dado que el AV y el CLA correlacionaron positivamente $(\mathrm{r}=0,85)$ y no se ha informado ninguna asociación de riesgo para el AV (Hodgson, et al., 1996) ni para el par AV/CLA (Roy, et al., 2007), estos parámetros deberán ser mejorados posiblemente mediante la inclusión adicional de una dosis baja de aceites poliinsaturados de girasol o soja.

En este trabajo, la relación CLA/AV (producto/precursor) resultó de 0,63 en la LE (Tabla 2), con una ligera disminución en la LCLA, pero manteniendo un valor alto $(0,58)$. El resultado fue mayor al rango $(0,39-0,46)$ observado por Gagliostro et al. (2012) ante el suministro de dosis crecientes del suplemento a vacas lecheras de raza Holstein. Esto permite inferir que la actividad de la $\Delta-9$ desaturasa mamaria (generadora del CLA a partir del AV) fue adecuada en las vacas de ambos lotes. Puesto que dicha relación fue mayor al valor promedio de $0,33 \%$ informado por otros autores para vacas suplementadas con AGPI (Antonacci, et al., 2017; Griinari y 
Bauman, 1999) puede inferirse que la biodisponibilidad del AV pudo haber sido limitante.

Al igual que en Gagliostro et al. (2017), la concentración del ácido linoleico (cis-9 cis-12 C18:2) fue incrementada desde un valor basal de $1,92 \mathrm{~g} / 100 \mathrm{~g}$ en la LE a $2,51 \mathrm{~g} / 100$ $\mathrm{g}$ en la LCLA. Esos valores se encuentran dentro del rango normal de concentración (2-3\%) informado por Chilliard et al. (2007). El incremento porcentual en la LCLA del ácido linoleico (30,83\%) respecto a la LE resultó superior al 19,69\% observado en un trabajo previo (Gagliostro, et al., 2017). Estos incrementos sugieren cierta protección del compuesto (liberación ruminal atenuada) que no resulta deseable para la generación del AV y del CLA y para mantener una baja relación omega-6/omega-3 en la leche.

En la LE, la concentración del cis-9, cis-12, cis-15 C18:3 o ácido $\alpha$-linolénico $(0,29 \mathrm{~g} / 100 \mathrm{~g})$ estuvo dentro del rango $(0,28-0,33 \mathrm{~g} / 100 \mathrm{~g})$ registrado en un ensayo precedente ( $\mathrm{Ga}$ gliostro, et al., 2013). Este AG de la serie omega 3 es esencial y de gran importancia en la salud humana. Por lo tanto, el fuerte aumento $(32,64 \%)$ en la concentración basal de este compuesto en la LCLA (Tabla 2) resulta relevante y comparable al obtenido en un ensayo precedente (Gagliostro, et al., 2013) con el mismo producto a igual dosis $(0,32-0,39 \mathrm{~g} / 100$ $\mathrm{g}$ de $\alpha$-linolénico). Esto podría indicar una acción protectora del DHA contenido en el suplemento utilizado sobre la biohidrogenación del ácido linolénico (AbuGhazaleh, et al., 2009; Chilliard, et al., 2000). Es de interés saludable ya que se trata de un AG esencial, precursor del EPA y del DHA, y de gran importancia en la salud humana y de la piel.

En el hombre, estudios epidemiológicos y experimentales han demostrado que los ácidos de la serie omega-3 presentan propiedades hipocolesterolémicas, antitrombóticas, antiinflamatorias e inmunosupresoras (Daviglus, et al., 1997; Calder, 2006, 2008 y 2012). Los AG omega-3 (a-linolénico, EPA y DHA) presentan un mayor grado de insaturación, lo que mejora la fluidez y la diversidad de las estructuras en las que participan. Esto facilita la función de "comunicación celular" y un correcto funcionamiento del tejido nervioso y la retina, potenciando la inteligencia, la memoria y las facultades mentales en general. Los reguladores derivados de los ácidos grasos omega-3 atenúan los efectos de los omega- 6 y son eficaces en la prevención de problemas cardiovasculares (efecto antiaterogénico, hipotensor (menor al de los AG n-6) $\mathrm{y}$ antitrombótico), y en la reducción de la incidencia de tumores y de procesos inflamatorios y degenerativos crónicos. Por lo tanto, es importante mantener una adecuada relación entre el consumo de AG omega-6/omega-3 (entre 10:1 a 5:1 o menor) para asegurar un buen equilibrio entre los reguladores perjudiciales (omega-6) y los moderados (omega-3).

En la LE, la relación omega-6/ omega-3 resultó de 6,67, mientras que en la LCLA alcanzó un valor similar de 6,59 sin diferencias entre ambas leches ( $p<0,57$, Tabla 2$)$. En vacas lecheras suplementadas con una combinación de aceite de soja y lino (75:25) se obtuvieron relaciones omega-6/omega-3 de 5,6 en la leche (Antonacci, et al., 2017). Puesto que en la dieta habitual de los humanos la relación está entre 10 y 30 puede considerarse que estas leches contribuyen a reducir dicho parámetro.

Finalmente, la presencia del ácido oleico (cis-9 C18:1) fue significativamente superior $(+24,43 \%)$ en la LCLA respecto a la LE (Tabla 2). Este compuesto presente en la llamada "dieta mediterránea" resulta abundante en el aceite de oliva, con efectos beneficiosos sobre el perfil de lípidos sanguíneos y factores de riesgo de enfermedades cardiovasculares (Mensink, et al., 2003). Se ha descripto que los AG monoinsaturados modulan la presión sanguínea, mejoran la sensibilidad a la insulina y regulan los niveles de glucosa circulantes (Gillingham, et al., 2011). El aumento del ácido oleico luego del aporte de AGPI es un hecho bien documentado (Glasser, et al., 2008; Chilliard, et al., 2009).

\section{Conclusiones}

La LCLA presentó diferencias en sus ácidos grasos constitutivos respecto a la LE. Estas diferencias mejoran su valor saludable a través de una menor concentración de grasa saturada e índice aterogénico, con un aumento simultáneo de ácidos grasos saludables como el CLA natural, el oleico y el linolénico, manteniendo una adecuada relación omega-6/ omega-3. El aumento en la concentración del ácido vaccénico deberá mejorarse por sus propiedades saludables intrínsecas y por ser el precursor de CLA a nivel mamario. Las razones que explican la baja respuesta en $\mathrm{AV}$ podrían estar asociadas al modo de suministro del producto, a algún componente desconocido de la ración con efectos inhibidores sobre las bacterias involucradas, a una liberación ruminal lenta y/o subóptima del ácido linoleico, a desvíos hacia la producción de trans-10 C18:1 en alimentación estabulada o a una combinación de estos factores. Estos aspectos merecen evaluarse experimentalmente.

Los resultados permitieron obtener una leche de mayor valor saludable para consumo directo o para elaboración de productos lácteos, pero la magnitud del incremento del CLA deberá mejorarse.

\section{Reconocimientos}

Este trabajo fue parcialmente financiado por la Agencia Nacional de Investigación e Innovación (ANII), proyecto Leche Talar CLA, Res. $\mathrm{N}^{\circ}$ 2484-017, y por el Instituto Nacional de Tecnología Agropecuaria (INTA). Agradecemos al personal de Tambo Talar por la colaboración en el manejo de los animales experimentales.

\section{Referencias}

AbuGhazaleh, A. y Jenkins, T. C., 2004. Short communication: decosahexaenoic acid promotes vaccenic acid accumulation in mixed rumen cultures when incubated with linoleic acid. En: Journal Dairy Sci., 87, pp.1047-1050.

AbuGhazaleh, A.A., Potu, R.B. y Ibrahim, S., 2009. Short communication: the effect of substituting fish oil in dairy cow diets with docosahexaenoic acid-micro algae on milk composition and fatty acids profile. En: Journal of Dairy Science, 92(12), pp.6156-6159.

Antonacci, L.E., Gagliostro, G.A., Cano, A.V. y Bernal, C.A., 2017. Effects of feeding combinations of soybean and linseed oils on productive performance and milk fatty acid profile in grazing dairy cows. En: Agricultural Sciences, 8, pp. 984-1002. https://doi.org/10.4236/as.2017.89072

Awad, A.B., Hermann, T., Fink, C.S. y Horvath, P.J., 1995. 18:1 n7 Fatty acids inhibit growth and decreased inositol phosphate release in HT-29 cells compared to n-9 fatty acids. En: Cancer Lett., 91, pp.55-61. 
Banni, S., Angioni, E., Murru, E., Carta, G., Melis, M.P., Bauman, D., Dong, Y. y Ip, C., 2001. Vaccenic acid feeding increases tissue levels of conjugated linoleic acid and suppreses development of premalignant lesions in rat mammary gland. En: Nutrition and Cancer, 41, pp.91-97. https://doi.org/10.1080/01635581.2001.9680617

Bargo, F., Muller, L.D., Kolver, E.S. y Delahoy, J.E., 2003. Invited review: production and digestion of supplemented dairy cows on pasture. En: Journal of Dairy Science, 86, pp.1-42.

Bhattacharyaa, A., Banua,J., Rahmana, M., Causeyb J. y Fernandes, G., 2006. Biological effects of conjugated linoleic acids in health and disease. En: Journal of Nutritional Biochemistry, 17, pp.789-810.

Calder, P.C., 2006. n-3 Polyunsaturated fatty acids, inflammation, and inflammatory Diseases. En: American Journal of Clinical Nutrition, 83 (Suppl 1), pp.505S-19S.

Calder, P.C., 2008. Fatty acids and inflammation from the membrane to the nucleus and from the laboratory bench to the clinic. En: Clinical Nutrition, 2. pp.5-12.

Calder, P.C., 2012. Fatty acids and inflammatory processes: nutrition or pharmacology? En: British Journal of Clinical Pharmacology, 75 (3), pp. 645-662.

Chilliard, Y., Ferlay, A., Mansbridge, R.M. y Doreau, M., 2000. Ruminant milk fat plasticity: nutritional control of saturated, polyunsaturated, trans and conjugated fatty acids. En: Annales de Zootechnie, 49, pp.181-205. https://doi.org/10.1051/animres:2000117

Chilliard, Y. y Ferlay, A., 2004. Dietary lipids and forages interactions on cow and goat milk fatty acid composition and sensory properties. En: Reprod. Nutr. Dev., 44, pp. 467-492.

Chilliard, Y., Glasser, G., Enjalbert, F., Ferlay, A., Bocquier, F. y Schimidely, P.H., 2007. Conferencia: resultados recientes sobre los efectos de la alimentación en la composición en ácidos grasos de la leche de vaca, cabra y oveja. En: Revista Argentina de Producción Animal, 27(3), pp.197-213.

Chilliard, Y., Martin, C., Rouel, J. y Doreau, M. 2009. Milk fatty acids in dairy cows fed whole crude linseed, extruded linseed, or linseed oil, and their relationship with methane output. En: Journal of Dairy Science, 92, pp.5199-5211.

Daviglus, M.L., Stamler, J., Orencia, A.J., Dyer, A.R., Liu, K., Greenland. P., Walsh, M.K., Morris, D. y Shekelle, R.B., 1997. Fish consumption and the 30 -year risk of fatal myocardial infarction. En: New England Journal of Medicine, 336, pp.1046-1053. DOI: 10.1056/ NEJM19970410336150.

Dhiman, T.R., Nam, S.H., Ure, AL., 2005. Factors affecting conjugated linoleic acid content in milk and meat. En: Crit. Rev. Food Sci. Nutr., 45, pp.463-82.

Gagliostro, G. A. y Chilliard, Y., 1992. Utilización de lípidos protegidos en la nutrición de vacas lecheras. Efectos sobre la producción y composición de leche, y sobre la ingestión de materia seca y energía. En: Revista Argentina de Producción Animal, 12(1), pp.1-15.

Gagliostro, G.A., Antonacci, L., Espiñeira, M., Williner, M.R. y Bernal, C.A., 2013. Suplementación con ácidos grasos poliinsaturados y perfil de ácidos grasos en vacas lecheras. En: Revista Argentina de Producción Animal, 33, (Supl. 1).

Gagliostro, G.A., Antonacci, L.E., Ballistreri, J., Bonina, E., Williner, M.R. y Bernal, C.A., 2012. Effects of a solid oil supplement (Oralac) on milk fatty acid composition of grazing dairy cows. En: Journal of Animal Science, 90, Suppl. 3/Journal of Dairy Science, 95, Suppl. 2., pp.119.
Gagliostro, G.A., Garciarena, D.A., Rodriguez, M.A. y Antonacci, L.E., 2017. Feeding polyunsaturated supplements to grazing dairy cows improve the healthy value of milk fatty acids. En: Agricultural Sciences, 8, pp.759-782. https://doi.org/10.4236/as.2017.88057.

Gillingham, L.G., Harris-Janz, S. y Jones, P.J., 2011. Dietary monounsaturated fatty acids are protective against metabolic syndrome and cardiovascular disease risk factors. En: Lipids, 46, pp.2019-228.

Glasser, F., Ferlay, A. y Chilliard, Y., 2008. Oilseed lipid supplements and fatty acid composition of cow milk: a Meta-Analysis. En: Journal of Dairy Science, 91, pp.46874703.

Griinari, J.M. y Bauman, D.E., 1999. Biosynthesis of conjugated linoleic acid and its incorporation into meat and milk in ruminants. En: $M$. P. Yurawecz, M. M. Mossoba, J. K. G. Kramer, M. W. Pariza and G. J. Nelson, ed. Advances in conjugated linoleic acid research. Champaign: AOCS Press, Champaign. pp.180-200.

Grummer, R.R., 1991. Effect of feed on the composition of milk fat. En: Journal of Dairy Science, 74, pp.3244-3257.

Ha, Y.L., Storkson J. y Pariza, M.W., 1990. Inhibition of benzo(a) pyreneinduced mouse forestomach neoplasia by conjugated dienoic derivatives of linoleic acid. En: Cancer Research, 50, pp.1097-1101.

Hodgson, J.M., Wahlqvist, M.L., Boxall, J.A. y Balazs, N.D., 1996. Platelet trans fatty acids in relation to angiographically assessed coronary artery disease. En: Atherosclerosis, 120, pp.147-154.

Houseknecht, K.L., Vanden Heuvel, J.P, Moya-Camarena, S.Y., et al., 1998. Dietary conjugated linoleic acid normalizes impaired glucose tolerance in the Zucker diabetic fatty fa/ fa rat. En: Biochemical and Biophysical Research Commun, 244, pp.678-682.

Huang, Y., Schoonmaker, J.P., Bradford, B.J. y Beitz, D.C., 2008. Response of milk fatty acid composition to dietary supplementation of soy oil, conjugated linoleic acid, or both. En: Journal of Dairy Science, 91, pp.260-270.

Ip, C., Banni, S., Angioni, E., Carta, G., McGinley, J., Thompson, H.J., Barbano, D. y Bauman, D., 1999. Conjugated linoleic acid-enriched butter fat alters mammary gland morphogenesis and reduces cancer risk in rats. En: J. Nutr., 129, pp.2135-2142.

Larson, S.C, Bergkvist, L. y Wolk, A., 2005. Hight-fat dairy food and conjugated linoleic acid intakes in relation to colorectal cancer incidence in the Swedish Mammography cohort. En: American Journal of Clinical Nutrition, 82, pp.894-900.

Lee, K.N., Kritchevsky, D. y Pariza, M.W., 1994. Conjugated linoleic acid and atherosclerosis in rabbits. En: Atherosclerosis, 108, pp.19-25.

Livingstone, K.M., Lovegrove, J.A. y Givens, D.I., 2012. The impact of substituting SFA in dairy products with MUFA or PUFA on CVD risk: evidence from human intervention studies. En: Nutrition Research Reviews, 25(2), pp.193-206. doi:10.1017/S095442241200011X.

Masson, L.T., Alfaro, C., Camilo, A., Carvalho, P., Illesca, R., Torres, M., Tavares do Carmo, J. Mancini-Filho y Bernal, C.A., 2015. Fatty acid composition of soybean/sunflower mix oil, fish oil and butterfat applying the AOCS Ce $1 \mathrm{j}-07$ method with a modified temperature program. En: Grasas y Aceites, 66, pp.e064. ISSN-L: 0017-3495. doi: http://dx.doi.org/10.3989/gya.0692141). 
Mensink, R.P., Zock, P.L., Kester, A. D. y Katan, M.B., 2003. Effects of dietary fatty acids and carbohydrates on the ratio of serum total to HDL cholesterol and on serum lipids and apolipoproteins: a meta analysis of 60 controlled trials. En: American Journal of Clinical Nutrition, 77, pp.1146-1155.

Miller, C.C., Park, Y., Pariza, M.W. y Cook, M.E., 1994. Feeding conjugated linoleic acid to animals partially overcomes catabolic responses due to endotoxin injection. En: Biochemical and Biophysical Research Communication, 198, pp.1107-1112.

Palmquist, D. L., Beaulieu, A. D. y Barbano, D. M., 1993. ADSA foundation symposium: Milk fat synthesis and modification. Feed and animal factors influencing milk fat composition. En: Journal of Dairy Science, 76, pp.1753-1771.

Palmquist, D. y Griinari, M., 2006. Milk fatty acid composition in response to reciprocal combinations of sunflower and fish oils in the diet. En: Animal Feed Science and Technology, 131, pp.358-369.

Parodi, P.W., 2003. Conjugated linoleic acid in food. En: Sébédio, J.L., Christie, W.W., Adloff, R., eds. Advances in conjugated linoleic acid in food. Vol. 2. Illinois: AOCS Press. pp.101-122.

Piperova, L.L., Teter, B.B., Bruckental, I., Sampugna, J. Mills S.,E., Yurawecz,M.P., Fritsche, J., Ju, K. y Erdman, R.A., 2000. Mammary lipogenic enzyme activity, trans fatty acids and conjugated fatty acids are altered in lactating dairy cows fed a milk-fat depressing diet. En: Journal of Nutrition, 130, pp.2568-2574.

Roy, A., Chardigny, J.M., Bauchart, D., Ferlay, A., Lorenz, S., Durand, D., Gruffat, D., Faulconnier, Y., Sébedio, J.M. y Chilliard, Y. 2007. Butters rich either in trans-10-C18:1 or in trans-11-C18:1 plus cis-9, trans-11 CLA differentially affect plasma lipids and aortic fatty streak in experimental atherosclerosis in rabbits. En: Animal, 1, pp.467-476.

Ryder, J.W., Portocarrero, C.P., Song, X.M, et al., 2001. Isomerspecific antidiabetic properties of conjugated linoleic acid. Improved glucose tolerance, skeletal muscle insulin action, and UCP-2 gene expression. En: Diabetes, 50, pp.1149-1157.

Schrezenmeir, J. y Jagla, A., 2000. Milk and diabetes. En: Journal of the Animal College of Nutrition, 19(2), pp.176:190.
Schroeder, G.F., Gagliostro, G.A., Bargo, F., Delahoy, J.E. y Muller, L.D., 2004. Effects of fat supplementation on milk production and composition by dairy cows on pasture: a review. En: Livestock Production Science, 86(1-3), pp.1-18.

Siurana, A. y Calsamiglia, S., 2016. A metaanalysis of feeding strategies to increase the content of conjugated linoleic acid (CLA) in dairy cattle milk and the impact on daily human consumption. En: Animal Feed Science and Technology, 217, pp.13-26.

Smit, L.A., Baylin, A. y Campos, H., 2010. Conjugated linoleic acid in adipose tissue and risk of myocardial Infarction. En: American Journal of Clinical Nutrition, 92(1), pp.34-40.

Stanton, C., Murphy, J., McGrath, E. y Devery, R., 2003. Animal feeding strategies for conjugates linoleic acid enrichment of milk. En: Sébédio, J.L., Christie, W.W., Adloff, R., eds. Advances in Conjugated Linoleic Acid in Food. Vol 2. Illinois: AOCS Press. pp.123-145.

Turpeinen, A.M., Mutanen, M., Aro, A., Salminen, I., Basu, S., Palmquist, D.L. y Griinari, J.M., 2002. Bioconversion of vaccenic acid to conjugated linoleic acid in humans. En: American. Journal of Clinical Nutrition, 76, pp.504-510.

Ulbritch, T. L. V. y Southgate, D. A.T., 1991. Coronary heart disease: seven dietary factors. En: Lancet, 338, pp.985-992.

Watkins, B.A. y Li, Y., 2003. CLA in functional food: enrichment of animal products. En: Sébédio, J.L, Christie, W.W., Adloff, R., eds. En: Advances in conjugated linoleic acid in food. Vol 2. Illinois: AOCS Press. pp.174-188.

Wu Z. y Huber, J.T., 1994. Relationship between dietary fat supplementation and milk protein concentration in lactating cows: A review. En: Livestock Production Science, 39, pp.141-155.

Yang M. y Cook, M.E., 2003. Dietary conjugated linoleic acid decreased cachexia, macrophage tumor necrosis factoralpha production, and modifies splenocyte cytokines production. En: Experimental Biological Medicine, (Maywood), 228, pp.51-58.

Zheng, H.C., Liu, J.X., Yao, J.H., Yuan, Q., Ye, H.W., Ye, J.A. y Wu, Y.M., 2005. Effects of dietary sources of vegetable oils on performance of high- yielding lactating cows and conjugated linoleic acids in milk. En: J. Dairy Sci., 88, pp.2037-2042. 